\title{
Maize meal fortification is associated with improved vitamin A and iron status in adolescents and reduced childhood anaemia in a food aid-dependent refugee population
}

\author{
Andrew Seal ${ }^{1, *}$, Emmanuel Kafwembe ${ }^{2}$, Ismail AR Kassim ${ }^{1}$, Mei Hong ${ }^{1}$, \\ Annie Wesley ${ }^{3}$, John Wood ${ }^{5}$, Fathia Abdalla ${ }^{4}$ and Tina van den Briel ${ }^{5}$ \\ ${ }^{1}$ Centre for International Health and Development, Institute of Child Health, 30 Guilford Street, London WC1N \\ 1EH, UK: ${ }^{2}$ Tropical Disease Research Centre, Ndola, Zambia: ${ }^{3}$ Micronutrient Initiative, Ottawa, Canada: \\ ${ }^{4}$ United Nations High Commissioner for Refugees, Geneva, Switzerland: 5 World Food Programme, Rome, Italy
}

Submitted 27 April 2007: Accepted 16 October 2007: First published online 21 December 2007

\begin{abstract}
Objective: To assess changes in the Fe and vitamin A status of the population of Nangweshi refugee camp associated with the introduction of maize meal fortification.

Design: Pre- and post-intervention study using a longitudinal cohort.

Setting: Nangweshi refugee camp, Zambia.

Subjects: Two hundred and twelve adolescents (10-19 years), 157 children (6-59

months) and 118 women (20-49 years) were selected at random by household survey in July 2003 and followed up after 12 months.

Results: Maize grain was milled and fortified in two custom-designed mills installed at a central location in the camp and a daily ration of $400 \mathrm{~g}$ per person was distributed twice monthly to households as part of the routine food aid ration. During the intervention period mean $\mathrm{Hb}$ increased in children $(0.87 \mathrm{~g} / \mathrm{dl} ; P<0.001)$ and adolescents $(0.24 \mathrm{~g} / \mathrm{dl} ; \quad P=0.043)$ but did not increase in women. Anaemia decreased in children by $23.4 \%(P<0 \cdot 001)$ but there was no significant change in adolescents or women. Serum transferrin receptor ( $\log _{10}$-transformed) decreased by $-0.082 \mu \mathrm{g} / \mathrm{ml}(P=0.036)$ indicating an improvement in the Fe status of adolescents but there was no significant decrease in the prevalence of deficiency $(-8.5 \%$; $P=0 \cdot 079)$. In adolescents, serum retinol increased by $0.16 \mu \mathrm{mol} / 1(P<0 \cdot 001)$ and vitamin A deficiency decreased by $26 \cdot 1 \%(P<0 \cdot 001)$.

Conclusions: The introduction of fortified maize meal led to a decrease in anaemia in children and a decrease in vitamin A deficiency in adolescents. Centralised, camp-level milling and fortification of maize meal is a feasible and pertinent intervention in food aid operations.
\end{abstract}

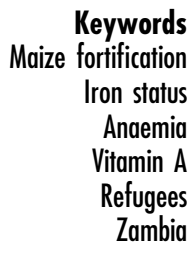

Globally, micronutrient malnutrition continues to affect many populations and exerts a negative impact on health, mortality and human capital. Populations affected by emergencies may be especially vulnerable due to preexisting low intakes of micronutrients and a dependence on international food aid, the micronutrient content of which has been documented to be frequently inadequate $^{(1,2)}$. This makes such populations prone to developing deficiency diseases, particularly in refugee crises that have become protracted ${ }^{(3-7)}$

Improving the micronutrient content of the food basket can be achieved in various ways, including the promotion and facilitation of home gardens, income generation and improving market access ${ }^{(8)}$. However, the constraints of the physical and political environment often mean that the food aid ration has to supply the bulk of nutrient requirements, and this is typically done through the supply of a cereal staple, pulses, fortified blended foods, vegetable oil, salt, and some other commodities. Even so, general rations often remain deficient in micronutrients and fortification of the staple commodity is one potentially cost-effective approach to closing the nutrient gap. Staple food fortification may also be attractive for population targeting purposes and the risk of excessive nutrient intake is low compared with the supply of small quantities of highly fortified commodities.

Micronutrient fortification of staple cereals has many advantages and has been associated with improvements in micronutrient status in many different contexts ${ }^{(9)}$. Recently, the fortification of maize meal has be shown to be effective in improving nutritional status in infants and young African children, and international guidelines are 
available on implementation from the $\mathrm{WHO}^{(10-13)}$. However, in practice it is difficult to ensure that commodities are appropriately fortified, the fortificant levels are suitable for the population's needs, shelf-life constraints can be overcome, and the required supplies can be made available at the onset of emergency operations and sustained throughout. This is a particular concern in populations consuming maize, as the shelf-life of milled whole maize is reduced to a matter of weeks due to the rapid onset of fat rancidity at tropical temperatures and humidity.

To combat some of these problems, custom mobile milling and fortification equipment was developed that allowed for the production of fortified maize meal at the level of the refugee camp ${ }^{(14)}$. This technical development work was undertaken as part of a collaborative pilot project involving the United Nations World Food Programme (WFP), United Nations High Commissioner for Refugees (UNHCR), CARE International, Natural Resource Institute and others, and was funded by Micronutrient Initiative, Canada and WFP through a grant from the Canadian International Development Agency. The resulting equipment was field-tested in Nangweshi refugee camp, Zambia, beginning in 2003.

Nangweshi refugee camp was opened in 2000 in response to the influx of refugees fleeing the Angolan civil war. It is located in the Western Province of Zambia, about $180 \mathrm{~km}$ from the border with Angola. The population of the camp came mainly from the southern and eastern parts of Angola. The population figure for June 2003 was recorded by UNHCR as 26061 with 8404 households. A range of international non-government organisations and UN agencies provided services in the camp during the intervention period.

The planned ration supplied by the WFP comprised maize meal, pulses, vegetable oil and salt. Based on the planned rations, an initial analysis of the food aid basket was performed to assess the likely prevalence of micronutrient deficiencies ${ }^{(15)}$. This showed that the ration was deficient in a number of micronutrients including vitamin $\mathrm{A}$, $\mathrm{Fe}$, riboflavin and vitamin $\mathrm{C}$ when compared against WHO safe levels of intake for emergency-affected populations ${ }^{(16)}$. High-dose vitamin A capsules were distributed periodically to children in the camp and $\mathrm{Fe} /$ folate tablets were available for pregnant women via the antenatal care programme. The present paper reports the evaluation of the impact of fortified maize meal distribution on anaemia in children, adolescents and women, and on Fe deficiency and vitamin A deficiency in adolescents.

\section{Methods}

\section{Sampling method}

The study was a pre- and post-intervention design using a longitudinal cohort with measurements conducted at two time points, July 2003 and July 2004. It was not possible to include a control group owing to the geographical layout of the camp and logistic, operational and ethical considerations. The sample size was calculated using the EpiInfo $^{\mathrm{TM}}$ software package version 6.04 (Centers for Disease Control and Prevention, Atlanta, GA, USA) so as to be able to detect a reduction of 14 percentage points from an assumed pre-intervention anaemia and vitamin A deficiency prevalence of $40 \%$, with an $\alpha$ risk of $5 \%, 80 \%$ power, and assuming $10 \%$ refusal or loss to follow-up. This resulted in a sample size of 210 adolescents (10-19 years), who were our primary subject group. All children (6-59 months) and women ( $20-49$ years) belonging to the adolescent's households were also sampled.

One hundred and fifty households were selected using a systematic random sampling method with houses as the sampling unit ${ }^{(16)}$. The sampling interval was determined based on population figures obtained from UNHCR and the total number of households obtained from CARE International, who was responsible for camp management. Using these data, the average household size and the appropriate sampling intervals were determined. A random number was then drawn between 1 and the sampling interval. This determined the first household to survey. Subsequent households were selected by adding the sampling interval to the current house number. This ensured equal probability of selection of households and individuals. Teams were supplied with printed lists of the houses to be visited and included in the survey. The house list also contained columns to enable recording of any necessary re-visits and comments.

Within each house, all individuals of the appropriate age groups were interviewed and measured. If any refused or were absent then they were not replaced in the sampling plan. Subjects who were reported to be in health centres or hospitals were located for interviewing and measurement. If a dwelling was empty neighbours were asked about the normal occupants. If reliable information was obtained, eligible individuals from within these 'empty' households were included in the sample frame and not replaced. Re-visiting to locate absent household members was undertaken up to two times. Subjects who had sickle cell anaemia diagnosed in the first survey were excluded from the longitudinal follow-up study. All questionnaire, anthropometry and biological sample collections were performed at the household level.

Collection of age data was based, where possible, on vaccination or health record cards, or subject or parental recall. For children whose age could not be determined, a height range of $65-110 \mathrm{~cm}$ was used to identify children aged 6-59 months $^{(16)}$.

\section{Staff training and data collection}

Three separate survey teams were used consisting of three or four local medical and nutrition staff. All team members attended $2 \mathrm{~d}$ of training on the selection 
of individuals to be sampled, administration of questionnaires, the importance of universal safety precautions, collection of blood samples, use of the Hemocue, and detection of clinical signs of micronutrient deficiency diseases. A $2 \mathrm{~d}$ refresher training was conducted prior to starting the second survey.

\section{Etbical approval}

Ethical approval for the surveys was obtained from the Research Ethics Committee of the Tropical Disease Research Centre, Ndola, Zambia, and a letter of support issued by the ethical review board of the Institute of Child Health, London. Before survey initiation, community leaders were consulted to discuss any questions and reservations they had about the study. Individual informed consent was obtained from all participants before samples were taken. No material benefits, other than feedback on their nutritional and health situation, were offered to encourage participation. It was stressed that refusal to participate would have no adverse affects on entitlements to food aid or other benefits. Any subjects found to be suffering from malnutrition or infections were referred to the camp health facilities using local referral criteria.

\section{Distribution of fortified maize meal}

Each household received a $400 \mathrm{~g}$ ration of $97 \%$ extraction, fortified maize meal per registered refugee, irrespective of age, during the duration of the intervention period, which lasted from November 2003 until June 2004. The ration supplied by the WFP also included, per person per day, $120 \mathrm{~g}$ pulses (beans or peas), $20 \mathrm{~g}$ vegetable oil and $10 \mathrm{~g}$ salt. The maize grain included in the pre-intervention ration was replaced by the fortified maize meal during the period of the intervention. Table 1 shows the fortificant formulation that was added to the maize meal, which was then blended, bagged and stored for a maximum of 4 weeks prior to distribution. Distributions took place every two weeks at a central location where a representative from each household received the ration that was scooped from storage sacks by CARE International staff. Food basket monitoring (on-site distribution monitoring) was undertaken by nutrition and health staff working for African Humanitarian Action.

\section{Dietary assessment}

Calculation of the energy and nutrient content of the distributed food aid ration was performed using the NutVal 2006 spreadsheet application (UCL Centre for International Health and Development/UNHCR/WFP, London, UK) ${ }^{(15)}$. Information on the frequency of consumption of food items, during the previous seven days, was collected by interviewing women and children or caregivers. Fourteen food items were selected for inclusion in the questionnaire following interviews with key informants and focus group discussions. Average consumption of each item was
Table 1 Micronutrient fortificant added to $1 \mathrm{~kg}$ of maize meal

\begin{tabular}{|c|c|}
\hline Vitamin $A(\mu \mathrm{g} R)$ & 2100 \\
\hline Thiamin $(\mathrm{mg})$ & $4 \cdot 4$ \\
\hline Riboflavin (mg) & $2 \cdot 6$ \\
\hline Nicotinamide (mg) & $35 \cdot 0$ \\
\hline Vitamin $\mathrm{B}_{6}(\mathrm{mg})$ & $2 \cdot 5$ \\
\hline Vitamin $B_{12}(\mu \mathrm{g})$ & $10 \cdot 0$ \\
\hline Folic acid (mg) & 1.5 \\
\hline $\mathrm{Fe}^{\star}(\mathrm{mg})$ & $35 \cdot 0$ \\
\hline Znt (mg) & $20 \cdot 0$ \\
\hline
\end{tabular}

$\mathrm{RE}$, retinol equivalents.

*Elemental.

tAs $\mathrm{ZnO}$.

calculated and a Food Variety Score (FVS) was determined for each individual from the number of unique food items consumed during the recall period ${ }^{(17)}$.

\section{Antbropometry}

Anthropometric measurements were taken on children in each household using standard anthropometric equipment and methods ${ }^{(16)}$. Anthropometry was not conducted on adolescents and women due to lack of resources for suitable equipment. Weight was measured to the nearest $100 \mathrm{~g}$ and height or length to the nearest millimetre. Children less than 24 months old were measured lying down, while children aged 24-59 months were measured standing. Global acute malnutrition was defined as a weight-for-height $Z$ score $<-2$ compared with the National Center for Health Statistics/WHO growth reference curves $^{(18)}$ or oedema, and severe acute malnutrition was defined as a weight-for-height $Z$ score $<-3$ or oedema. Chronic malnutrition (stunting) was defined by using the same $Z$ score cut-offs for the height-for-age index. Bipedal pitting oedema was assessed by placing thumb pressure on to both feet of children and infants for a period of $3 \mathrm{~s}$. After releasing pressure the presence or absence of an indent was noted.

\section{Hb measurement}

Measurement of $\mathrm{Hb}$ was performed in the household using a portable Hemocue Photometer (HemoCue AB, Angelholm, Sweden), utilising the azidemethaemoglobin principle. Peripheral blood was collected from a finger stick made using a safety lancet (Hemocue). The altitude of the camp is about $900 \mathrm{~m}$ above sea level, so no altitude adjustment to the $\mathrm{Hb}$ cut-off was required. The $\mathrm{Hb}$ concentration cut-offs used to define anaemia were: $<110 \mathrm{~g} / 1$ for children aged $6-59$ months; $<115 \mathrm{~g} / 1$ for adolescents aged 10-11 years; <120 g/l for adolescents aged 12-14 years; $<120 \mathrm{~g} / 1$ for adolescent females aged 15-19 years; $<130 \mathrm{~g} / \mathrm{l}$ for adolescent males aged 15-19 years; <120 g/1 for women aged $20-45$ years.

\section{Malaria blood films}

Thick and thin smears were made from peripheral blood collected directly onto the same slide, using standard 
methods. The slide was air dried, packed and transported to the field laboratory for analysis. Slides were stained using the forward and reverse field stain technique for thick and thin films, respectively. Thin films were fixed prior to staining with absolute alcohol. The thick blood film was examined using the Greenwood and Armstrong method. This method gives the approximate number of parasites per microlitre of blood by counting the average number of parasite pigments in fifty oil immersion fields and multiplying by $500^{(19)}$.

\section{Sickle cell anaemia testing}

During the pre-intervention survey thin blood films were screened for the presence of cell abnormalities suggestive of sickle cell anaemia. The Meta-bisulphite Test (Sickle Cell Slide Test) was used as a confirmatory test on suspect films. Sickling of abnormal cells was produced by placing a drop of capillary blood onto a slide and adding two equal drops of a freshly prepared solution of $2 \%(\mathrm{w} / \mathrm{v})$ sodium metabisulphite. The mixture was mixed with the corner of the cover slip, cover-slipped, sealed with wax and allowed to stand for $30 \mathrm{~min}$. The slide was then examined for sickle cells at power $10 \times$ and $40 \times$. If initially negative, the slide was incubated for a further hour and regarded as negative if no sickle cells were present after this extended incubation ${ }^{(20)}$.

\section{Collection of serum for biochemical analyses}

Approximately $200 \mu \mathrm{l}$ of peripheral blood was collected from the same or a second finger stick into a Microtainer serum separation tube (Becton Dickinson, New Jersey, USA). This was then labelled and stored on ice in a vaccine container at $4-8^{\circ} \mathrm{C}$ until centrifugation at the end of the day. Following centrifugation, the serum was transferred to a screw top cryovial and frozen at $-20^{\circ} \mathrm{C}$. Samples were subsequently transported on wet ice to the Tropical Disease Research Centre laboratory in Ndola, Zambia.

\section{Measurement of serum transferrin receptor}

Analysis of serum transferrin receptor (sTfR) was performed on serum using a sandwich ELISA kit (catalogue no. TF-94) purchased from Ramco Laboratories (Stafford, TX, USA). Survey samples were analysed in two separate batches, and the CV for the high and normal value quality control samples was $9 \cdot 4 \%(n$ 32) and $7 \cdot 3 \%(n$ 17), respectively. Concentrations $>8.3 \mathrm{mg} / \mathrm{l}$ were used to define Fe deficiency. Fe-deficiency anaemia was defined in subjects with both an abnormal sTfR and an abnormal $\mathrm{Hb}$ concentration.

\section{Measurement of serum retinol}

Serum retinol in adolescents was measured by HPLC ${ }^{(21)}$. Serum samples were thawed and mixed by inverting the tube several times. A $50 \mu$ l aliquot of serum was placed in a screw-capped glass test-tube and $50 \mu$ l of ethanolic retinyl acetate was added as internal standard. The retinol in the sample was then extracted twice using $200 \mu \mathrm{l}$ n-hexane. The hexane in the extract was evaporated under $\mathrm{N}_{2}$ to leave a residue containing the retinol. The residue was dissolved in $50 \mu \mathrm{l}$ of dichloromethane-propanol solution (4:1, by vol.) and $10 \mu \mathrm{l}$ was analysed using a reversed-phase column and a methanol-water mixture (98:2, by vol.) as the mobile phase. Retinol was detected by monitoring the absorption at $325 \mathrm{~nm}$. Samples were analysed in two separate batches and the CV for pooled quality control serum samples was $4 \cdot 8 \%$ ( $n$ 139) and $4 \cdot 1 \%$ ( $n$ 89) for the high and low values, respectively. Subjects with serum retinol concentrations of $0 \cdot 7-0.35 \mu \mathrm{mol} / 1$ were classified as being at medium risk and those with concentrations $<0.35 \mu \mathrm{mol} / 1$ at high risk for adverse effects. The lower limit of detection for the analytical system used was $0 \cdot 11 \mu \mathrm{mol} / \mathrm{l}$. Samples with values less than $0 \cdot 11 \mu \mathrm{mol} / 1$ ( $n 3$ in the pre-intervention survey) were recorded as $0 \cdot 11$.

\section{Collection of urine and baematuria testing}

During the pre-intervention survey, teams collected urine from consenting adolescents in $100 \mathrm{ml}$ collection cups. The urine was then transferred from the collection cups into a $10 \mathrm{ml}$ urine collection tube (Sarstedt Monovette) and labelled with the identification number of the adolescent. Urine tubes were stored in plastic bags at $0-8{ }^{\circ} \mathrm{C}$ until the end of the day, when samples were tested for the presence of haematuria using a dipstick (Combur10Test; Roche, Basel, Switzerland) as a proxy measure for Schistosoma haematobium egg infestation.

\section{Data entry and analysis}

Data were entered and analysed using EpiInfo ${ }^{\mathrm{TM}}$ version 6.04 d (CDC), Excel 2000 (Microsoft Corporation, Redmond, WA, USA) and the Statistical Package for the Social Sciences version $12 \cdot 0 \cdot 1$ (SPSS Inc., Chicago, IL, USA) software packages. Correlations between normally distributed continuous variables were tested using Pearson's test and the Wilcoxon signed ranks test was used for nonnormally distributed variables.

Paired, two-tailed $t$ tests were conducted to assess changes in means between pre- and post-intervention measures and $\chi^{2}$ tests were used to assess changes in proportions. To control for potential confounding, an SPSS Generalised Linear Model ANOVA repeated measures (RM) technique was used in which the intervention was evaluated as a within-subject factor and potential confounders were entered as covariates if they were continuous variables or as between-subject factors if they were categorical variables ${ }^{(22)}$. Pre- and post-intervention changes in continuous variables were expressed as estimated marginal means. Significance level was taken at the $P<0.05$ level. 


\section{Results}

Figure 1 illustrates the passage of subjects through the study. Household sampling identified 216 eligible adolescents who were invited to participate. There were no refusals and all individuals were found. However, two were later identified as being outside the desired age range during data checking and data were missing for one subject. Data were analysed on the remaining 213. One hundred and seventy-six adolescents were found and followed up in the post-intervention survey. Different sample sizes reported for subsequent laboratory analyses reflect a failure to complete analysis where inadequate sample volumes were obtained.

One hundred and fifty-seven children were identified as eligible for recruitment. No refusals were received and all subjects were located. However, two subjects were excluded and dropped from the study following the diagnosis of sickle cell anaemia. Pre-intervention data were analysed from the remaining 155 .

A total of 118 women were found to be eligible and recruited to the study. Two were absent from the household at the time of the survey and could not be found but there were no refusals. Pregnant women were excluded from the analysis of $\mathrm{Hb}$ concentrations, as this is affected by gestational age. Complete pre-intervention data were therefore collected on ninety-one women. Successful follow-up was achieved for over $80 \%$ of adolescents and children but for only $64 \%$ of women. The main cause for loss to follow-up in adolescents and children was movement of subjects out of the camp due to repatriation to Angola. In women, repatriation and pregnancy were both important causes.

The characteristics of the population sample at recruitment are given in Table 2. Measurement of all parameters in the different population groups was not possible owing to resource constraints. The anthropometric status of the children regarding wasting was good, with no children having $Z$ score below -2 ; however, $44.5 \%$ were classified as stunted (height-for-age $Z$ score below -2). Malaria parasitaemia was present in less than $10 \%$ of subjects and, in positive cases, the median levels were low at 175 and 210 parasites/ $\mu$ l for children and adolescents, respectively. Low concentration haematuria was found in adolescents but was detectable by dipstick only.

Food frequency data from the pre-intervention survey indicated that maize, received as food aid, was consumed twice a day on average by both adolescents and children. Consumption frequency did not alter when this was replaced by fortified maize meal during the intervention period. While no quantitative intake data were collected, observations of food preparation and cooking practices indicated that maize formed a substantive proportion of food intake. These observations were consistent with the calculation that maize meal contributed $71 \%$ of the dietary energy supplied by the food aid ration ${ }^{(15)}$. (a)

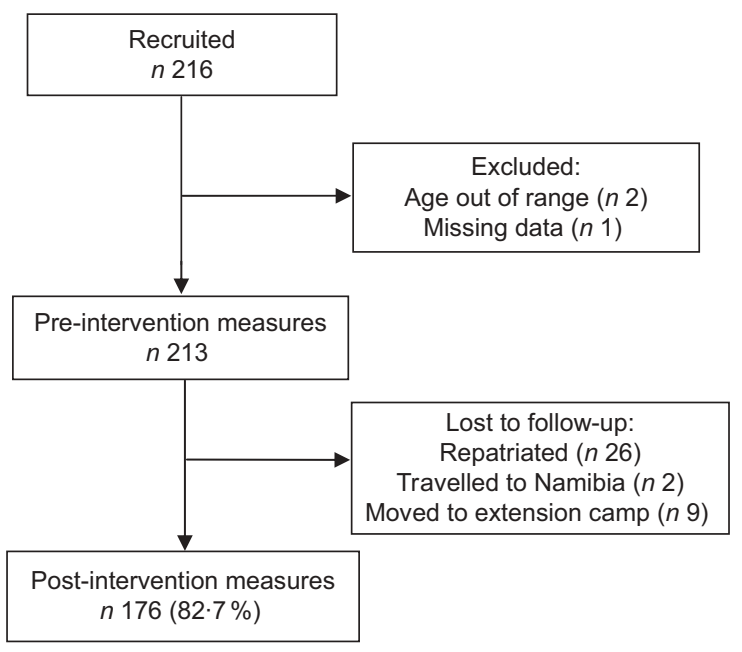

(b)
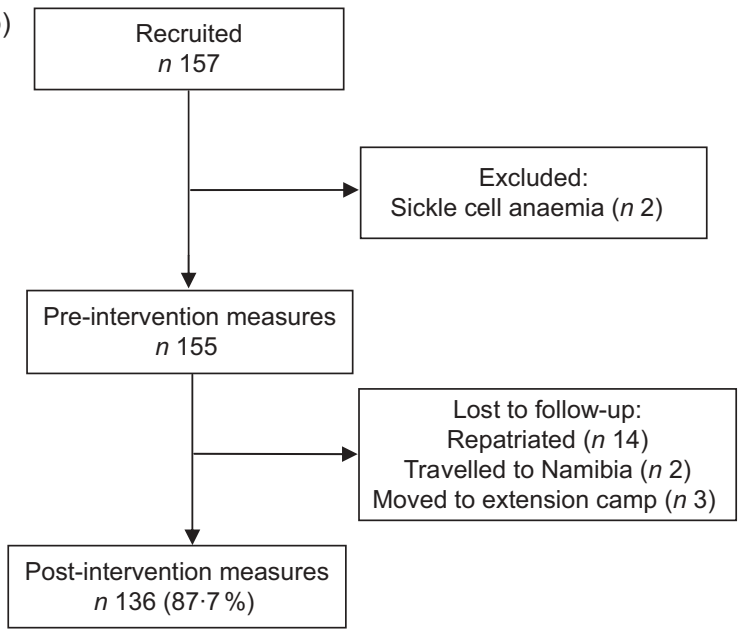

(c)

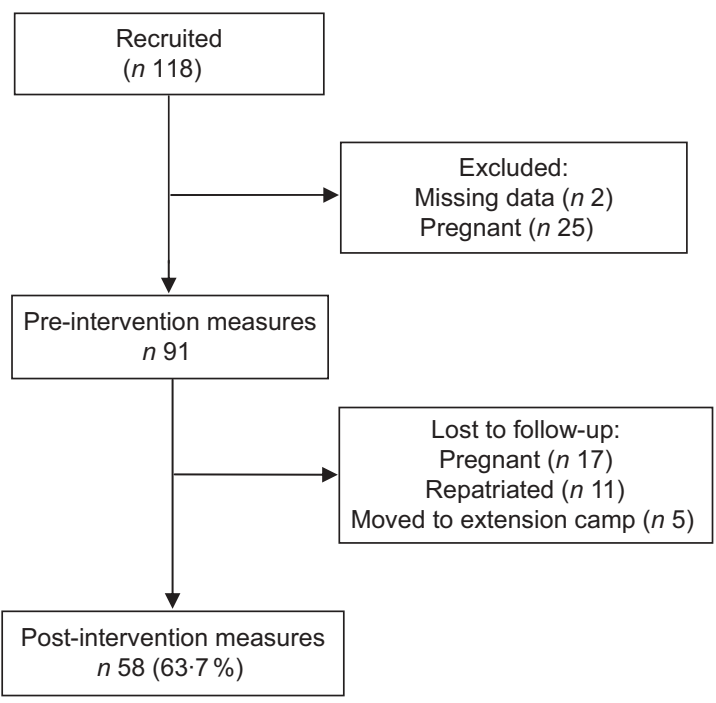

Fig. 1 Study flow chart showing recruitment and follow-up for (a) adolescents, (b) children and (c) women. The percentage of each cohort successfully followed up is given in parentheses 
Table 2 Characteristics of the population sample at recruitment, Nangweshi refugee camp, Zambia

\begin{tabular}{|c|c|c|c|c|c|c|}
\hline & \multicolumn{6}{|c|}{ Population group } \\
\hline & \multicolumn{2}{|c|}{ Adolescents ( $n$ 213) } & \multicolumn{2}{|c|}{ Children (n 155) } & \multicolumn{2}{|c|}{ Women ( $n$ 91) } \\
\hline & Mean & $95 \% \mathrm{Cl}$ & Mean & $95 \% \mathrm{Cl}$ & Mean & $95 \% \mathrm{Cl}$ \\
\hline Age $^{*}$ & $13 \cdot 4$ & $10,19+$ & $32 \cdot 8$ & $6,59+$ & $32 \cdot 8$ & $20,48 \dagger$ \\
\hline$\%$ male & $51 \cdot 2$ & & $44 \cdot 5$ & & - & \\
\hline WHZ & ND & & $-0 \cdot 14$ & $-0.27,-0.01$ & ND & \\
\hline $\mathrm{HAZ}$ & ND & & $-1 \cdot 83$ & $-2 \cdot 01,-1 \cdot 64$ & ND & \\
\hline Slide positive malaria (\%) & $7 \cdot 0$ & $4 \cdot 0,11 \cdot 3$ & $9 \cdot 0$ & $5 \cdot 0,14 \cdot 7$ & ND & \\
\hline Haematuria (\%) & $5 \cdot 8$ & $2 \cdot 4,12 \cdot 6$ & ND & & ND & \\
\hline FVS & $3 \cdot 27$ & $3.04,3.50$ & 3.08 & $2 \cdot 79,3 \cdot 37$ & ND & \\
\hline
\end{tabular}

WHZ, weight-for-height $Z$ score; HAZ, height-for-age $Z$ score; FVS, Food Variety Score; ND, not determined.

*Mean age measured in years for adolescents, months for children and years for women.

tRange.

Table 3 Changes in $\mathrm{Hb}$ concentration and anaemia prevalence following the distribution of fortified maize meal, Nangweshi refugee camp, Zambia

\begin{tabular}{|c|c|c|c|c|c|c|c|c|}
\hline & \multicolumn{4}{|c|}{$\mathrm{Hb}(\mathrm{g} / \mathrm{dl})$} & \multicolumn{4}{|c|}{ Anaemia (\%) } \\
\hline & Pre & Post & $\Delta^{*}$ & $P$ & Pre & Post & $\Delta$ & $P+$ \\
\hline \multicolumn{9}{|c|}{ Adolescents } \\
\hline$n$ & 213 & 176 & 176 & & 213 & 176 & & \\
\hline Mean & $12 \cdot 9$ & $13 \cdot 1$ & 0.24 & 0.043 & $19 \cdot 2$ & $24 \cdot 4$ & $5 \cdot 2$ & 0.216 \\
\hline $95 \% \mathrm{Cl}$ & $12 \cdot 7,13 \cdot 2$ & $12 \cdot 8,13 \cdot 3$ & $0.01,0.47$ & & $14 \cdot 2,25 \cdot 2$ & $18 \cdot 3,31 \cdot 5$ & & \\
\hline \multicolumn{9}{|l|}{ Children } \\
\hline$n$ & 155 & 136 & 136 & & 213 & 176 & & \\
\hline Mean & $10 \cdot 9$ & $11 \cdot 8$ & $0 \cdot 87$ & $<0.001$ & $47 \cdot 7$ & $24 \cdot 3$ & $-23 \cdot 4$ & $<0.001$ \\
\hline $95 \% \mathrm{Cl}$ & $10 \cdot 6,11 \cdot 2$ & $11 \cdot 6,12 \cdot 1$ & $0.28,0.92$ & & $39 \cdot 7,55 \cdot 9$ & $17 \cdot 3,34 \cdot 4$ & & \\
\hline \multicolumn{9}{|l|}{ Women } \\
\hline$n$ & 91 & 58 & 58 & & 91 & 58 & & \\
\hline Mean & $13 \cdot 1$ & $12 \cdot 6$ & -0.29 & $0 \cdot 193$ & $16 \cdot 5$ & $27 \cdot 6$ & $11 \cdot 1$ & $0 \cdot 104$ \\
\hline $95 \% \mathrm{Cl}$ & $12 \cdot 7,13 \cdot 5$ & $12 \cdot 1,13 \cdot 2$ & $-0 \cdot 15,0.74$ & & $9 \cdot 8,26 \cdot 1$ & $17 \cdot 1,41 \cdot 1$ & & \\
\hline
\end{tabular}

${ }^{*}$ Difference in estimated marginal means of paired measures, adjusted for age.

$+\chi^{2}$ test.

In children, a paired $t$ test ( $n$ 136) showed a significant increase in mean $\mathrm{Hb}$ of $0.9 \mathrm{~g} / \mathrm{dl}(P<0.001)$ between the pre-intervention and post-intervention surveys. As shown in Table 3, there was also a significant reduction in the prevalence of anaemia. However, $\mathrm{Hb}$ in children was correlated with age at baseline (Pearson correlation $r=0 \cdot 176 ; P=0 \cdot 028$ ) and also at follow-up. Hb was also correlated with height-for-age $Z$ score at baseline (Pearson correlation $r=0 \cdot 186 ; P=0 \cdot 021$ ) and follow-up. In contrast, FVS, sex, presence of slide positive malaria and weight-for-height $Z$ score were not associated with $\mathrm{Hb}$ concentrations at baseline or follow-up.

The RM technique was performed to control for possible confounding. As the difference in age between baseline and follow-up surveys was a constant for all subjects (one year), age could not be included as a covariate or between-subject factor in the RM analysis. At baseline, the relationship between age and $\mathrm{Hb}$ was described by a regression coefficient of $0 \cdot 267 \mathrm{~g} / \mathrm{dl}$ per year (95\% CI 0.029, 0.505). This value was therefore subtracted from the $\mathrm{Hb}$ concentration of all subjects at follow-up to adjust for increases due to age. The change in height-for-age $Z$ score ( $\triangle \mathrm{HAZ}$ ) was then included as a covariate in the RM analysis and the increase in the estimated marginal mean $\mathrm{Hb}$ remained significant.

In adolescents, RM analysis ( $n$ 176) showed a significant increase in mean $\mathrm{Hb}$ from $12 \cdot 9$ to $13 \cdot 1 \mathrm{~g} / \mathrm{dl}$ during the intervention but no significant change in the prevalence of anaemia. There was no correlation between age and $\mathrm{Hb}$ concentration and there was also no significant difference in $\mathrm{Hb}$ between boys and girls at baseline or follow-up. Fe status ( $\log _{10}$ sTfR) was highly correlated with $\mathrm{Hb}$ concentration (Pearson correlation $r=-0 \cdot 447 ; P<0 \cdot 001)$ but it was not controlled for as a confounder in the analysis of $\mathrm{Hb}$ change as it lies on the hypothesised causal pathway. $\mathrm{Hb}$ in adolescents was not correlated with FVS, the presence of slide positive malaria or haematuria. RM analysis ( $n$ 58) of women who were not pregnant at either time point showed no significant difference in mean $\mathrm{Hb}$ between baseline and follow-up.

Changes in the concentration of sTfR and the prevalence of Fe deficiency are shown in Table 4. There was no difference in mean $\log _{10}$ sTfR concentration or the prevalence of Fe deficiency according to sex at either 
Table 4 Changes in serum transferrin receptor (sTfR) concentration and prevalence of iron deficiency in adolescents following the distribution of fortified maize meal, Nangweshi refugee camp, Zambia

\begin{tabular}{|c|c|c|c|c|c|c|c|c|}
\hline & \multicolumn{4}{|c|}{$\log _{10} \operatorname{sTfR}(\mu \mathrm{g} / \mathrm{ml})$} & \multicolumn{4}{|c|}{$\%$ Fe deficient $(\mathrm{sTfR}>8.3 \mu \mathrm{g} / \mathrm{ml})$} \\
\hline & Pre & Post & $\Delta^{*}$ & $P$ & Pre & Post & $\Delta$ & $P+$ \\
\hline$n$ & 203 & 165 & 165 & & 203 & 165 & & \\
\hline Mean & 0.875 & 0.790 & -0.083 & 0.004 & $31 \cdot 5$ & $23 \cdot 0$ & $-8 \cdot 5$ & 0.079 \\
\hline $95 \% \mathrm{Cl}$ & $0.849,0.901$ & $0.733,0.845$ & $-0.139,-0.027$ & & $25 \cdot 2,38 \cdot 4$ & $16 \cdot 8,30 \cdot 2$ & & \\
\hline
\end{tabular}

*Difference in estimated marginal means of paired measures.

$+\chi^{2}$ test.

Table 5 Changes in serum retinol concentration prevalence of vitamin A deficiency in adolescents following the distribution of fortified maize meal, Nangweshi refugee camp, Zambia

\begin{tabular}{|c|c|c|c|c|c|c|c|c|}
\hline & \multicolumn{4}{|c|}{ Serum retinol $(\mu \mathrm{mol} / \mathrm{l})$} & \multicolumn{4}{|c|}{$\%$ vitamin $A$ deficient (serum retinol $<0.7 \mu \mathrm{mol} / \mathrm{l}$ ) } \\
\hline & Pre & Post & $\Delta^{*}$ & $P$ & Pre & Post & $\Delta$ & $P+$ \\
\hline$n$ & 207 & 172 & 172 & & 207 & 172 & & \\
\hline Mean & 0.73 & 0.94 & $0 \cdot 162$ & $<0.001$ & $46 \cdot 4$ & $20 \cdot 3$ & $-26 \cdot 1$ & $<0.001$ \\
\hline $95 \% \mathrm{Cl}$ & $0.69,0.77$ & $0.89,0.98$ & $0 \cdot 120,0 \cdot 204$ & & $39 \cdot 4,53 \cdot 4$ & $14 \cdot 6,27 \cdot 1$ & & \\
\hline
\end{tabular}

*Difference in estimated marginal means of paired measures, adjusted for age.

$+\chi^{2}$ test.

baseline or follow-up. In the 165 adolescents who had test results available at both time points, the median STfR concentration decreased between baseline and follow-up from $7 \cdot 2$ to $6 \cdot 2 \mu \mathrm{g} / \mathrm{ml}$. There was no association between STfR concentration and age or presence of slide positive malaria. However, FVS was negatively correlated with STfR at baseline (but not follow-up) indicating that better diet diversity was associated with improved Fe status during the pre-intervention survey (Pearson correlation with $\log _{10}$ sTfR $r=-0 \cdot 190 ; P=0 \cdot 027$ ).

$\mathrm{RM}$ analysis performed on the $\log _{10}$-transformed sTfR values, with the inclusion of the change in FVS ( $\Delta$ FVS $)$ as a covariate, indicated a significant decrease in sTfR during the intervention period. Fe deficiency decreased in adolescents from a prevalence of $31.5 \%$ at baseline to $23.0 \%$ at follow-up. However, this decrease was not statistically significant.

The mean levels of serum retinol are given in Table 5 . There was no significant difference in mean retinol concentration between males and females but there was a strong correlation with age. Linear regression analysis showed that retinol concentration increased by an average of $0.048 \mu \mathrm{mol} / 1$ per year (95\% CI $0 \cdot 038,0 \cdot 059)$ for each year of age between 10 and 19 years ( $n$ 207). This age dependence was also seen at follow-up. Vitamin A concentrations were also correlated with $\mathrm{Hb}$ and sTfR concentrations at baseline but not at follow-up. However, vitamin A concentration was not affected by the presence of slide positive malaria at baseline or follow-up, and was not affected by FVS.

To adjust for the effect of age, $0 \cdot 048 \mu \mathrm{mol} / \mathrm{l}$ was subtracted from the post-intervention measures before analysis using RM. Change in sTfR and change in $\mathrm{Hb}(\Delta \mathrm{sTfR}$, $\Delta \mathrm{Hb}$ ) were included in the model as covariates. During the intervention period there was a significant increase in retinol concentration and, as shown in Table 5, a significant reduction in the prevalence of vitamin A deficiency. Although age was associated with baseline retinol concentration, it did not interact with the intervention effect $(P=0 \cdot 808)$.

\section{Discussion}

These data show a clear association between the introduction of fortified maize meal and an improvement in the Fe and vitamin A status of a food aid-dependent refugee population. Improvements in the status of adolescents and a reduction in anaemia in children are important public health gains. While no impact was observed in women, the small sample size and low statistical power achieved for this group leaves the finding open to question. Micronutrient deficiencies often occur in combination and while we collected no information on other deficiencies it is likely that the study population was deficient in a number of other micronutrients ${ }^{(23)}$. In particular, vitamin B deficiency is frequently found in maizeconsuming populations and health staff in the camp reported isolated clinical cases of pellagra during the intervention period $^{(24)}$. Niacin and other B vitamins were included in the maize meal fortificant and it is reasonable to suppose that consumption of the fortified maize meal may have led to improvements in population micronutrient status.

The strategy of staple foods fortification has been successful in improving the nutrition of populations in industrialised and developing countries ${ }^{(9)}$. Recent studies on maize meal fortification in Africa have shown positive 
results in reducing anaemia and in improving serum retinol concentrations and weight gain in young children $^{(11,13)}$. The present study is consistent with these findings on anaemia in children and is the first report of potential impact in adolescents. In any fortification programme the fortificant needs to be designed so as to maximise the beneficial nutritional impact while minimising risk of excessive ingestion by population subgroups with a high consumption of the food vehicle ${ }^{(25)}$. In the present programme the fortificant formulation was specified by Micronutrient Initiative and WFP in consultation with the Government of Zambia, and was based on previous WFP specifications, Zambian legislation and the latest findings from the literature.

The fortificant premix utilised elemental $\mathrm{Fe}$ as its $\mathrm{Fe}$ source; this appears to have been effective in increasing the Fe status of adolescents and was associated with a marked reduction in anaemia in children. However, other forms of Fe such as ferrous fumarate offer a much higher bioavailability, and the impact on Fe status may have been higher if they had been used instead of the elemental form ${ }^{(26)}$.

While fortification of food aid commodities is not new, the method employed here was unique and shows potential for replication in other food aid operations. Milling and fortification equipment was custom-designed and manufactured in a modular format and then shipped to the camp location where it was installed and operated at a centralised location. The production staff were members of the refugee population while programme management was undertaken by a non-government organisation (CARE International). The centralised location of the production site meant that supervision and quality control of production was more easily achieved. Samples of fortified material were regularly taken and tested for Fe concentration as a proxy marker for all the premix components.

Unfortunately, a cluster-randomised design or control group could not be employed in the present study owing to operational constraints and the geographical arrangement and proximity of the population housing. Extensive discussions during the planning phase indicated that to try and include control groups would have led to operational difficulties that could have threatened the successful implementation of the programme. The evidence of plausibility provided by this study therefore consists of association only, although extensive efforts were made to control for potential confounding factors ${ }^{(27)}$.

The data were analysed using an ANOVA RM procedure that allows for the analysis of repeated measurements on the same individual, the inclusion of potential confounders as covariates, and the generation of modeladjusted means (estimated marginal means). For the present study, where a paired analysis of longitudinal data in the absence of a control group was required, the technique was extremely useful.
Pre-intervention, the FVS was not associated with nutritional status expect for sTfR concentration in adolescents. FVS increased during the intervention and was not associated with age, suggesting a general improvement in access to a variety of foods. However, RM analysis showed that this improvement in food variety did not explain the improvements in Fe status associated with the introduction of maize meal fortification.

Food aid distribution and receipts were closely monitored using a systematic sampling method (data not shown). This revealed that subjects received a continuous supply of maize meal and other commodities during the intervention period. While classified as food aid-dependent, some agricultural and income-generating activities were undertaken by the camp population. Crops grown included maize, groundnuts, cowpeas and cassava and produce from home gardens, but the yield was considered by UNHCR to be insignificant as a supplement to the general ration ${ }^{(28)}$. Passes could be obtained from the Government of Zambia Ministry of Home Affairs to enable the refugees to undertake piecework in the local area. However, there was no evidence of significant improvement in economic conditions during the intervention period and the refugees remained dependent on the food aid ration as the major source of their daily food intake.

These findings provide support to the view that food aid fortification is an effective means of reducing childhood anaemia and improving adolescent $\mathrm{Fe}$ and vitamin A status in highly vulnerable, aid-dependent populations. In this context, its impact on anaemia in women remains unclear. The data suggest that local level, centralised milling and fortification of staple foods should be promoted and their nutritional impact and cost-effectiveness investigated in other operational contexts.

\section{Acknowledgements}

The Micronutrient Initiative, Canada, funded the study through a grant from the Canadian International Development Agency (CIDA).

The field work was conducted by the Institute of Child Health in collaboration with African Humanitarian Action and CARE International. Additional assistance was provided by the WFP, UNCHR, CORD, and a number of other individuals, without whom the study would not have been possible.

A.S. designed the study, managed the field work and drafted the manuscript, E.K. was responsible for the biochemical laboratory analyses and provided comments on the manuscript, I.A.R.K. conducted the microscopy and other laboratory work and in the field, M.H. co-managed the field teams and data entry, A.W. provided detailed comments on the manuscript draft, J.W. was responsible for the design and installation of the 
milling and fortification plant and quality control of fortification, F.A. assisted in the set up of the field work and provided detailed comments on the manuscript draft, T.v.d.B. provided guidance on project design and commented on the manuscript draft.

\section{References}

1. Malfait P, Moren A, Dillon JC, Brodel A, Begkoyian G, Etchegorry MG, Malenga G \& Hakewill P (1993) An outbreak of pellagra related to changes in dietary niacin among Mozambican refugees in Malawi. Int $J$ Epidemiol 22, 504-511.

2. Tomkins A \& Henry CJ (1992) Comparison of nutrient composition of refugee rations and pet foods. Lancet 340 , 367-368.

3. Toole MJ (1992) Micronutrient deficiencies in refugees. Lancet 339, 1214-1216.

4. Hassan K, Sullivan KM, Yip R \& Woodruff BA (1997) Factors associated with anemia in refugee children. J Nutr 127, 2194-2198.

5. Blanck HM, Bowman BA, Serdula MK, Khan LK, Kohn W \& Woodruff BA (2002) Angular stomatitis and riboflavin status among adolescent Bhutanese refugees living in southeastern Nepal. Am J Clin Nutr 76, 430-435.

6. Kemmer TM, Bovill ME, Kongsomboon W, Hansch SJ, Geisler KL, Cheney C, Shell-Duncan BK \& Drewnowski A (2003) Iron deficiency is unacceptably high in refugee children from Burma. J Nutr 133, 4143-4149.

7. Seal AJ, Creeke PI, Mirghani Z, Abdalla F, McBurney RP, Pratt LS, Brookes D, Ruth LJ \& Marchand E (2005) Iron and vitamin A deficiency in long-term African refugees. $J$ Nutr 135, 808-813.

8. Prinzo ZW \& De Benoist B (2002) Meeting the challenges of micronutrient deficiencies in emergency-affected populations. Proc Nutr Soc 61, 251-257.

9. Darnton-Hill I \& Nalubola R (2002) Fortification strategies to meet micronutrient needs: successes and failures. Proc Nutr Soc 61, 231-241.

10. Andang'o PEA, Osendarp SJM, Ayah R, West CE, Mwaniki DL, De Wolf CA, Kraaijenhagen R, Kok FJ \& Verhoef H (2007) Efficacy of iron-fortified whole maize flour on iron status of schoolchildren in Kenya: a randomised controlled trial. Lancet 369, 1799-1806.

11. Faber M, Kvalsvig JD, Lombard CJ \& Benade AJ (2005) Effect of a fortified maize-meal porridge on anemia, micronutrient status, and motor development of infants. Am J Clin Nutr 82, 1032-1039.
12. Food and Agriculture Organization of the United Nations/ World Health Organization (2006) Guidelines on Food Fortification with Micronutrients. Geneva: FAO/WHO.

13. Nesamvuni AE, Vorster HH, Margetts BM \& Kruger A (2005) Fortification of maize meal improved the nutritional status of 1-3-year-old African children. Public Health Nutr 8, 461-467.

14. van den Briel $\mathrm{T} \&$ Webb P (2003) Fighting world hunger through micronutrient fortification programs. Food Tech 57, 44-47.

15. Seal AJ (2006) NutVal 2006: A Spreadsheet Application for the Planning, Calculation, and Monitoring of Food Aid Rations. London: UCL Centre for International Health and Development/WFP/UNHCR.

16. World Health Organization (2000) The Management of Nutrition in Major Emergencies. Geneva: WHO.

17. Torheim LE, Barikmo I, Parr CL, Hatloy A, Ouattara F \& Oshaug A (2003) Validation of food variety as an indicator of diet quality assessed with a food frequency questionnaire for Western Mali. Eur J Clin Nutr 57, 1283-1291.

18. Dibley MJ, Goldsby JB, Staehling NW \& Trowbridge FL (1987) Development of normalized curves for the international growth reference: historical and technical considerations. Am J Clin Nutr 46, 736-748.

19. Cheesbrough M (1998) District Laboratory Practice in Developing Counties. Cambridge: Cambridge University Press.

20. Cheesbrough M (2000) District Laboratory Practice in Developing Countries. Part 2. Cambridge: Cambridge University Press.

21. De Ruyter MG \& De Leenheer AP (1976) Determination of serum retinol (vitamin A) by high-speed liquid chromatography. Clin Chem 22, 1593-1595.

22. SPSS Inc (2003) SPSS Advanced Models ${ }^{\mathrm{TM}}$ 12.0. Chicago, IL: SPSS Inc.

23. Ramakrishnan U (2002) Prevalence of micronutrient malnutrition worldwide. Nutr Rev 60, S46-S52.

24. Hegyi J, Schwartz RA \& Hegyi V (2004) Pellagra: dermatitis, dementia, and diarrhea. Int J Dermatol 43, 1-5.

25. Fletcher RJ, Bell IP \& Lambert JP (2004) Public health aspects of food fortification: a question of balance. Proc Nutr Soc 63, 605-614.

26. Lynch SR (2005) The impact of iron fortification on nutritional anaemia. Best Pract Res Clin Haematol 18, 333-346.

27. Victora CG, Habicht JP \& Bryce J (2004) Evidence-based public health: moving beyond randomized trials. Am J Public Health 94, 400-405.

28. United Nations High Commissioner for Refugees (2003) Briefing note for the Joint UNHCR/WFP Mission 30/01/03. Nangweshi: UNHCR Nangweshi Field Office. 